\title{
ANFIS Based Methodology for Sign Language Recognition and Translating to Number in Kannada Language
}

\author{
https://doi.org/10.3991/ijes.v5i1.6682 \\ Ramesh M. Kagalkar \\ Dr. D Y Patil School of Engineering and Technology, Pune, Maharashtra, India \\ rameshvtu10@gmail.com \\ Dr. S.V Gumaste \\ R. H. Sapat College of Engineering, Nashik, Maharashtra, India \\ svgumastelgmail.com
}

\begin{abstract}
In the world of signing and gestures, lots of analysis work has been done over the past three decades. This has led to a gradual transition from isolated to continuous, and static to dynamic gesture recognition for operations on a restricted vocabulary. In gift state of affairs, human machine interactive systems facilitate communication between the deaf, and hearing impaired in universe things. So as to boost the accuracy of recognition, several researchers have deployed strategies like HMM, Artificial Neural Networks, and Kinect platform. Effective algorithms for segmentation, classification, pattern matching and recognition have evolved. The most purpose of this paper is to investigate these strategies and to effectively compare them, which can alter the reader to succeed in associate in nursing optimum resolution. This creates each, challenges and opportunities for signing recognition connected analysis.
\end{abstract}

Keywords—signing recognition, hidden markov model (HMM), artificial neural network (ANN)

\section{Introduction}

Sign languages are the fore most raw and natural sort of languages can be dated back to as early because the advent of the human civilization, once the primary theories of sign languages appeared in history. It's started even before the emergence of spoken languages and this has big selection of application. The analysis is motivated as a result of it's a large vary of application initial is that an indication system would be probably helpful in aiding communication between members of the deaf community and therefore the hearing community with the folk. The second is that the method of developing distance learning teaching help additionally it's helpful in medical field additionally it's terribly facilitate full in observation of patients In the gift analysis may be a model system for the automated recognition of language, supported a series of artificial neural networks, , HMM, ANFIS. This language used for developed a 
system that is helpful for a standard man and deaf and dumb individuals. A straight forward language with one hand has constant suggests that| everywhere the planet and means either "hi"e or "goodbye". Many of ad jaunt foreign countries while not knowing the official language of the visited country and still manage to perform communication mistreatment language. These examples show that language may be thought-about international and used the majority over the planet and convey correct info to any or all. Therefore during this technique we tend to used ANFIS primarily based classifier for the popularity of language.

This paper is organized as follows. In the section 2 to follow, we have provided a brief overview of related work. In section 3 describe the existing system overviews. The detailed description of proposed method sing ANFIS classifier discussed in section 4. In section 5 provides experimental results analysis and discussion. Finally conclusion of work is outlined.

\section{$2 \quad$ Related Work}

In the past, many methodology of gesture recognition additionally human computer interface (HCI) prompt like neural network, HMMs and Fuzzy systems however these take issue from one to a different in their models. A number of them area unit neural network, HMMs [1] and Fuzzy systems [2]. The past decades have witnessed two specific patterns for classes of linguistic communication recognition the primary system is classes into the electromechanically device like a glove primarily based system once this technique developed analysis has been restricted to tiny scale systems in a position of recognizing a minimum set of a symptom language. Christopher Lee and Yangsheng $\mathrm{Xu}$ [3] developed a glove-based gesture recognition system that was able to acknowledge fourteen of the letters from the hand alphabet, learn new gestures and able to update the model of every gesture within the system in on-line mode, with a rate of $10 \mathrm{~Hz}$. Over the years advanced glove devices are designed like the Sayre Glove, deft Hand Master and Power Glove [4].

The foremost flourishing commercially obtainable glove is far and away the VPL knowledge Glove, Fels and Hinton 1995a, Fels and Hinton 1995 Although sharing an equivalent name, authors and far of the same technology because the Glove-Talk system, Glove-TalkII takes a basically completely different approach to the mapping of hand gestures to speech. The initial Glove-Talk maps every gesture to one word. Glove-Talk II is based on a way finer grained approach during which options of the hand area unit mapped onto the articulate options which management the assembly of speech. American linguistic communication is that the language of selection for most deaf folks within the USA. It a part of the "deaf culture" and includes its own system of puns, inside jokes, etc. However, American sign language is one in every of the various sign languages of the planet. As associate in training English speaker would have hassle understanding someone speaking Japanese, a speaker of American sign language would have trouble understanding the linguistic communication of Sweden. ASL also has its own descriptive linguistics that's completely different from English. 
ASL consists of roughly 6000 gestures of common words with finger orthography accustomed communicate obscure words or correct nouns. Finger orthography uses one hand and 26 gestures to speak the twenty six letters of the alphabets. [5] Samples of signs shown in Figure. There are a unit few technologies already victimization vision primarily based analysis system. Example, Hand gesture recognition which victimization 'Vision-Based' approaches use solely the vision device (camera) for perceive musical conductivity action. This technique works by once the conductor uses only one-side hand and should within the read vary of camera. When the camera capture the image of hand gesture, the system extract the human hand region that is the region of interest (ROI) victimization the intensity color information. The system is obtained the motion speed and therefore the direction by pursuit the middle of gravity $(\mathrm{COG})$ of the hand region that provides the speed of any conducting time pattern [6]. For this Hand Gesture Recognition project, relies on Human-Computer Interaction $(\mathrm{HCI})$ technology. The computer will perform hand gesture recognition on yank linguistic communication (ASL) [7]. The system use MATLAB Toolboxes, neural network to perform the gesture recognition. It work by feed various types of hand gestures pictures then into neural network ${ }^{e e}$ and the system can train the network itself. Once the "neural network" is trained, this neural network" will perform multiples of hand gesture recognition of American sign language [8].

MacLean James [9] planned the employment of a back propagation neural network for recognition of gestures from a collection of metameric hand pictures. This technique showed promise within the field of language invariant teleconferencing. Loeding and Sarkar [10] developed a system that created use of Signees i.e. elements of signs that area unit gift in most occurrences, taken from videos. Extraction of Signs is finished victimization iterated conditional modes (ICM). The feature extraction side of image analysis seeks to spot inherent characteristics, or features of objects found at intervals a picture. These characteristics area unit accustomed describe the article, or attribute of the article, before the next task of classification. Shreenivasan and Geethapriya [11] developed a period of time system that has applications in video games. Extraction and agglomeration of key points is finished victimization rotation and scale invariant matching (RASIM) and k-harmonic means that techniques. Ghosh and Ari [12] developed a system for human different and augmentative communication.

Amitkumar Shinde and Ramesh M. Kagalkar [13] This paper presents an Automatic translation system for gesture of manual alphabets in Marathi sign language. It deals with images of bare hands, which allows the user to interact with the system in a natural way. System provides an opportunity for deaf persons to communicate with normal people without the need of an interpreter. We are going to build a systems and methods for the automatic recognition of Marathi sign language. The first step of this system is to create a database of Marathi Sign Language. Hand segmentation is the most crucial step in every hand gesture recognition system since if we get better segmented output, better recognition rates can be achieved. The proposed system also includes efficient and robust hand segmentation and tracking algorithm to achieve better recognition rates. 
Rashmi. B. Hiremath and Ramesh. M. Kagalkar [14] The proposed work presented in this paper going to recognize the hand gestures of sign language from input video stream of the signer and interprets into corresponding Hindi words and sentences. For this purpose, the proposed system uses the techniques of image processing and synthetic intelligence to fulfill the objective. To carry out this task it uses image processing techniques such as frame extraction, erosion, dilation, edge detection, blur elimination, noise elimination, wavelet transform, image fusion techniques to section shapes in videos. It additionally uses descriptors of elliptical Fourier for feature extraction of shape and also uses analysis for the purpose of the feature set reduction as well as optimization. Extracted features with corresponding Hindi text are stored in the database and compared with given input testing video of the signer by a trained unclear inference system. Rashmi. B. Hiremath and Ramesh. M. Kagalkar [15] the proposed method of implementation utilizes the image processing methods and synthetic intelligence strategies to get the goal of sign video recognition. To carry out the proposed task implementation it uses image processing methods such as frame analyzing based tracking, edge detection, wavelet transform, erosion, dilation, blur elimination, noise elimination, on training videos. It also uses elliptical Fourier descriptors called SIFT for shape feature extraction and most important part analysis for feature set optimization and reduction. For result analysis, this paper uses different category videos such as sign of weeks, months, relations etc. Database of extracted outcomes are compared with the video fed to the system as a input of the signer by a trained unclear inference system

Rashmi. B. Hiremath and Ramesh. M. Kagalkar [16] the proposed framework going to recognize the sign of gesture-based communication from a video stream of the signer and deciphers into relating words and sentences in Hindi. The proposed framework utilizes the image preparing strategies and synthetic intelligence procedures to get the target. To do the proposed task execution it utilizes picture handling strategies, for example, frame differencing based tracking, edge recognition, wavelet change, picture combination systems to area shapes in our videos. It moreover utilizes descriptors of elliptical Fourier for feature extraction of shape and also uses analysis for the purpose of feature set reduction as well as optimization. Extracted features are stored into the database and compared with given input video of the signer by a trained unclear inference system

Ramesh M. Kagalkar and Dr. S.V. Gumaste [17] this paper reviews the intensive state of the art in automatic recognition of continuous signs, from different languages, supported the information sets used, features computed, technique used, and recognition rates achieved. In this paper discover that, in the past, most work has been tired finger-spelled words and isolated sign recognition, but recently, there has been vital progress within the recognition of signs embedded briefly continuous sentences. Paper tend to conjointly realize that researchers are getting down addressing the necessary downside of extracting and integration non-manual data that is gift in face and head movement and present results from experiments integration of non-manual options. Ramesh M. Kagalkar, Dr. Nagaraj H.N and Dr. S.V Gumaste [18] this paper primarily consists of the popularity of well-defined signs supported a posture of the hand. Since human beings tend to differ in terms of size and shape the foremost diffi- 
cult drawback consists of the segmentation and also the correct classification of the information's gathered from the input image, captured byone or additional cameras. The aim of this paper is to indicate that techniques have with success been tested and employed in order to unravel the issues mentioned higher than yielding a strong and reliable static hand gesture recognition system.

Ramesh M. Kagalkar, Dr. Nagaraj H.N [19] the aim of sign language alphabets recognition is to provide an easy, efficient and accurate mechanism for automatic translation of static sign (determined by a certain configuration of hand) to textual version in kannada language. Problem Statement: The work presented in this paper goal to develop a system for automatic translation of static gestures of alphabets in kannada sign language. It maps letters, words and expression of a certain language to a set of hand gestures enabling an in individual to communicate by using hands gestures rather than by speaking. The system capable of recognizing sign language symbols can be used as a means of communication with hard of hearing people. It has been divided into two phases firstly, feature extraction phase which in turn uses histogram technique, Hough and Segmentation to extract hand from the static sign. Secondly classification phase uses neural network for training samples. Extreme points were extracted from the segmented hand using star skeletonization and recognition was performed by distance signature.

Ramesh M. Kagalkar and S.V Gumaste [20] in this paper introduced a continuous Indian sign language recognition system, wherever each the hands are used for playacting any gesture. Recognizing a sign language gestures from continuous gestures could be a terribly difficult analysis issue. This paper solves the problem using gradient based key frame extraction technique. These key frames are useful for splitting continuous language gestures into sequence of signs further as for removing uninformative frames. After splitting of gestures every sign has been treated as associate degree isolated gesture. Then features of pre-processed gestures are extracted using orientation histogram $(\mathrm{OH})$ with principal component analysis (PCA) is applied for reducing dimension of features obtained after $\mathrm{OH}$. From this analysis we tend to found that the results obtained from Correlation and Euclidian distance offers higher accuracy then alternative classifiers.

\section{Exiting Method}

Above technique used for recognition, however in step with once training 50 samples the error index in ANFIS is incredibly low that's why we tend to are attempting to implement a system of linguistic communication recognition by victimization ANFIS.

\subsection{Neural Network}

The neural network is additionally referred to as artificial neural network (ANN), is a man-made intelligent system that relies on biological neural network. Neural networks able to be trained to perform a specific operate by adjusting the values of the 
connections (weight) between these parts. [21,22] Fig.1 shows a neural network diagram, it is adjusted and trained so as the actual input ends up in a particular target output. Example at Figure, the network is adjusted, supported a comparison of the output and also the target till the network output is matched the target. Now a days, neural network are often trained to unravel several trouble some issues round-faced by individual and computer.

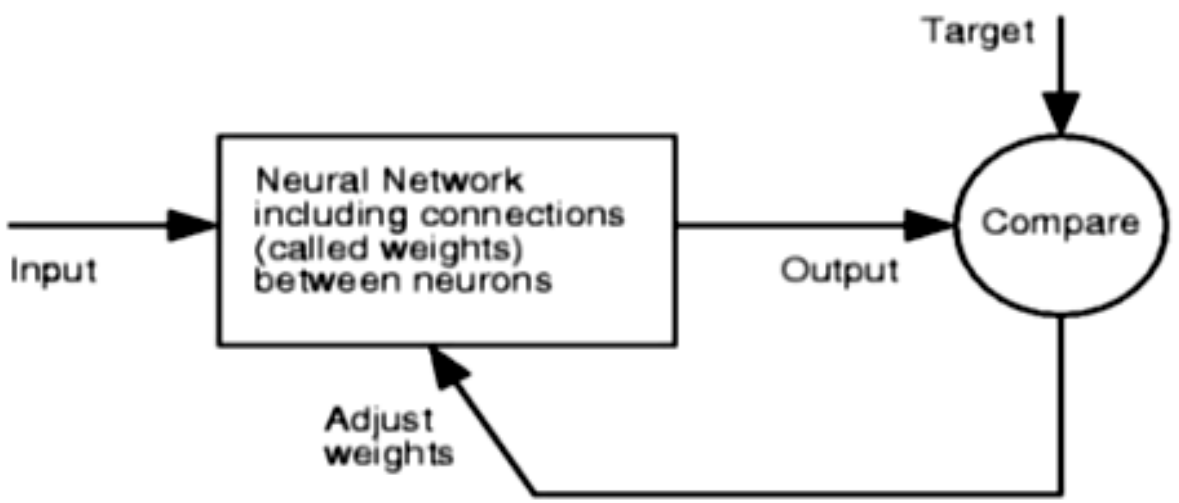

Fig. 1. Neural network block diagram.

\subsection{Hidden Marko Model}

In case of dynamic method modeling, the approach to be chosen is random. In nature, e.g. hidden mathematician models (HMMs) [23] or Dynamic Bayesian Networks [24]. A time domain method illustrates mathematician property if the contingent probability density of an occurrence, given all gift and past events, depends solely on the $\mathrm{j}^{\text {th }}$ most up-to-date event. If this event depends alone on the foremost recent past event, then the method is termed a primary order stochastic process. It's thoughtabout as a helpful assumption, whereas considering the orientations of hands of a sign alert for time axis. The HMM, far-famed for its wealthy mathematical structure, is taken into account to be a wide used tool for with efficiency modeling spatialtemporal data within the most natural method potential [25]. The algorithms which will be used within the pass embrace the Baum-Welch and Viterbi for analysis, learning, and decipherment before the interpretation will really be started upon The Hmm generalized topology is understood because the Ergodic model, as per Acharya's term conditions, whereby any state are often reached from the other state.

\section{Proposed Method Using ANFIS Classifier}

In proposed work we have given a system supported hand feature extraction together with a multi-layer fuzzy neural-network based mostly classifier. The hand 
gesture space is separated from the background by victimization skin detection and segmentation technique of coloring, then a contour of hand image are often used as a feature that describe the hand form. As such, the final method of the projected technique consists of three main parts,

\subsection{A Preprocessing Step}

This step is making a skin-segmented binary image by employing a threshold worth of chance, if the chance of a component in skin chance image is a lot of or capable calculable threshold worth, it supposed that this component represents coloring, if not it supposed that this component doesn't represent coloring. The coloring pixels are white and also the different ones are black within the skin metameric image.

\subsection{A Feature Extraction Step}

The hand contour can act because the feature of the gesture. The feature extraction side of image analysis seeks to spot inherent characteristics, or options of objects found inside a picture. These characteristics are accustomed describe the thing, or attribute of the thing, before the following task of classification. For posture recognition, (static hand gestures) options like fingertips, finger directions and hand's contours are often extracted. However such options don't seem to be continuously offered because of self-occlusion and lighting conditions. Feature extraction could be a complicated down side, and infrequently the full image or remodeled image is taken as input. Contour detection method consists of two steps, Initial realize the sting response in the slightest degree points in a picture victimization gradient computation and within the second step modulate the sting response at some extent by the response in its surround.

\subsection{A Classification Step}

The unknown gesture's feature are created and entered to the fuzzy neural network. The hand region obtained once the pre-processing stage Figure 2. The system model which will be used because the primary input file for the feature extraction step of the gesture recognition algorithmic program. Proposed classification method supported the employment of single, with hybrid training algorithmic program. Within the feature extraction stage the hand contour is resized so as to form it acceptable for neural network input, then it entered to the classification stage. The recognition method incorporates two phases, training and classification, as shown within the Figure 2. In figure 3 list of Sign symbol of numbers from 0 to 10 with Kannada meaning is listed. 


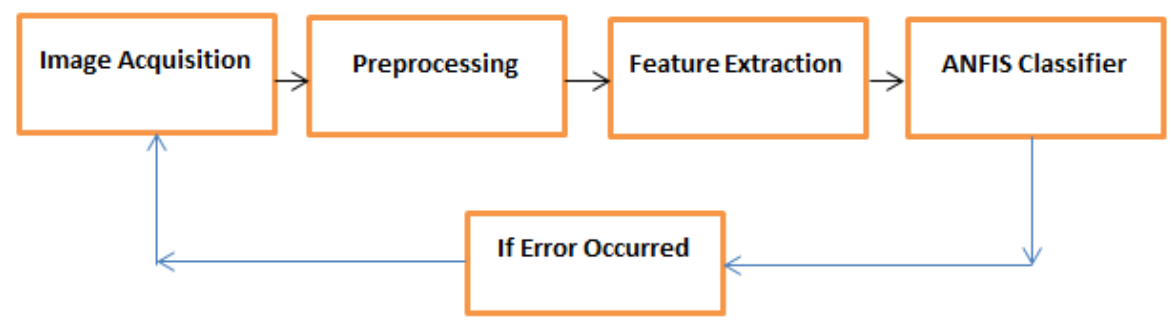

Fig. 2. Shows proposed system flow diagram.

Table 1. Shows the sign symbols meaning in Kannada as well as English is listed.

\begin{tabular}{|c|c|c|c|}
\hline Sr.No & Sign symbols & Kannada Numbers with words & Meaning in English \\
\hline 1. & & ( (సீอన్నె) & 0 \\
\hline 2. & & ( (ఒందు) & 1 \\
\hline 3. & & 9 (ఎరెడు) & 2 \\
\hline 4. & & 2 (మొలర) & 3 \\
\hline 5. & & 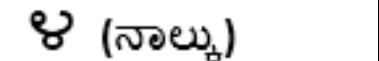 & 4 \\
\hline 6. & & भ่ (ఐదు) & 5 \\
\hline 7. & & e (ఆd) & 6 \\
\hline 8. & & 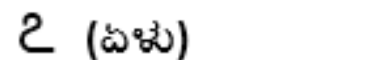 & 7 \\
\hline
\end{tabular}


Paper-ANFIS Based Methodology for Sign Language Recognition and Translating to Number in Kan..

\begin{tabular}{|c|c|c|}
\hline 9. & ల (ఎంఱు) & 8 \\
\hline 10. & ह (ఒంబత్సు) & 9 \\
\hline 11. & $00\left(\infty \overrightarrow{\omega_{2}}\right)$ & 10 \\
\hline
\end{tabular}

Numbers (0-10)

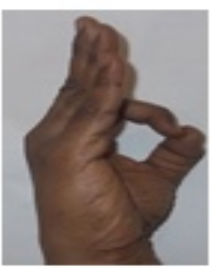

O

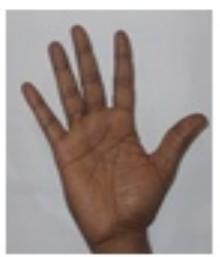

23

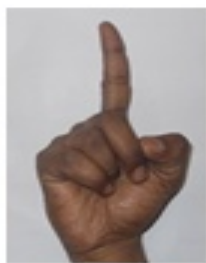

()

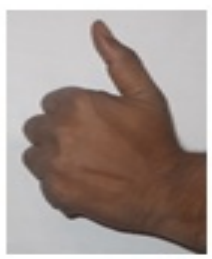

be

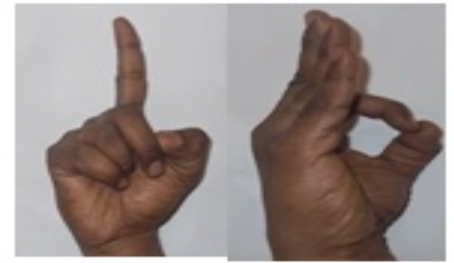

$\bigcirc \mathrm{O}$

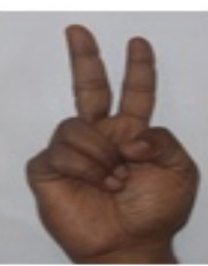

9)

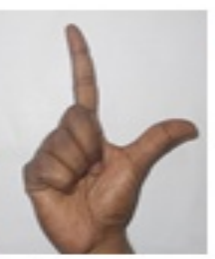

2

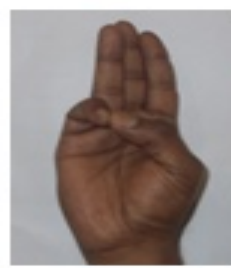

Q.

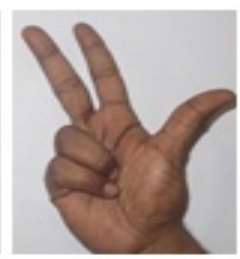

ej

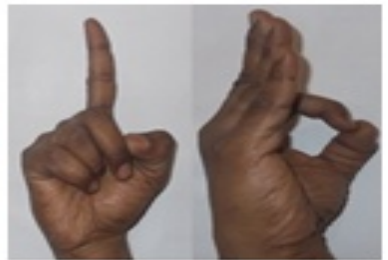

$\mathrm{O}$

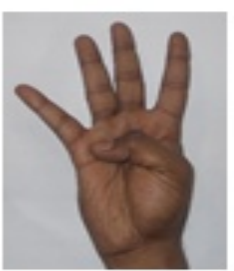

8

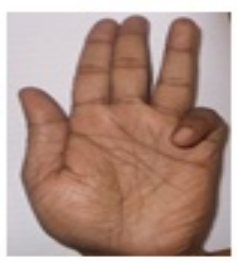

巨

Fig. 3. Shows Sign symbol of numbers from 0 to 10 . 


\section{$5 \quad$ Result Analysis and Discussion}

In this system we have a tendency to acknowledge language by exploitation three ways like Neural Network, PDIST and ANFIS. However the accuracy and time needed for classification is a smaller amount in ANFIS. The table 2 shows a table form of comparative analysis of accuracy and time needed for classification is given.

Table 2. Comparative analysis.

\begin{tabular}{|c|l|c|l|l|}
\hline Sr.No. & Classifier Algorithm & No. of Samples & Accuracy & $\begin{array}{c}\text { Time Required for Classifica- } \\
\text { tion }\end{array}$ \\
\hline 1. & PDIST & 35 & LOW & $0.0019099 \mathrm{~S}$ \\
\hline 2. & NN & 35 & HIGH & $0.0086336 \mathrm{~S}$ \\
\hline 3. & AFISN & 35 & HIGH & $0.000014 \mathrm{~S}==0 \mathrm{~S}$ \\
\hline
\end{tabular}

\section{Conclusion}

After the survey on the approaches utilized in varied vocabulary-based signing recognition systems, we are able to provide associate degree opinion concerning the methodologies and algorithms concerned. Most of the days, a mix of various strategies and algorithms should be accustomed succeed a moderate to acceptable rate of recognition. As an example some strategies square measure appropriate solely against dark back grounds. A system which supplies most potency, has low value, associate degreed is a best mixture of strategies, giving results against advanced backgrounds moreover, ought to be most popular. From a technical purpose of read, there's an enormous scope in future for analysis and implementation during this terribly field. The future years might witness a combinatorial explosion of various methodologies, like mistreatment many HMMs in parallel, freelance or coupled usage of ANN and HMMs etc. the final word gain of the projected study is big and accuracy and time needed for classification in ANFIS is a smaller amount than different therefore it's helpful to use in gesture recognition.

\section{$7 \quad$ References}

[1] Ww Lodzimierz Kasprzak, Artur Wilkowski and Karol Czapnik, "Hand Gesture Recognition in Image sequences Using Active Contours and HMMs" @inproceedings, Kasprzak 2009 HandGR, 2009.

[2] D. M. Gavrila, "The visual analysis of human movement: A survey." Computer Vision and Image Understanding, Elsevier, Volume 73, Issue 1, Pages 82-98, January 1999. https://doi.org/10.1006/cviu.1998.0716

[3] Liu Yucheng and Liu Yubin, "Incremental Learning Method of Least Squares Support Vector Machine", International Conference on Intelligent Computation Technology and Automation" VCL-94-104, 2010.

[4] K. T. Fang, Y.Wang, and P. M. Bentler, "Some applications of number theoretic methods in statistics," Stat. Sci., vol. 9, pp. 416-428, 1994. https://doi.org/10.1214/ss/1177010392 
Paper-ANFIS Based Methodology for Sign Language Recognition and Translating to Number in Kan...

[5] Gallaudet University Press (2004). 1,000 Signs of life: basic ASL for everyday conversation. Gallaudet University Press. http://venturebeat.com.

[6] [Bauer \& Hienz, 2000] "Relevant feature for video-based continuous sign language recognition." Department of Technical Computer Science, pages 440 - 445, Aachen University of Technology, Aachen, Germany, 2000.

[7] Starner, Weaver \& Pentland,"Real-time American Sign language recognition using a deskand wearable computer-based video." In proceedings IEEE transactions on Pattern Analysis and Machine Intelligence, pages 1371-1375, 1998.

[8] L. Fausett, "Fundamentals of Neural networks, architectures, algorithms, and applications", Prentice-Hall, Inc. 1994, pp-304-315.

[9] James MacLean, "Fast Hand Gesture Recognition for Real-Time Teleconferencing Applications, In International Workshop on Recognition, Analysis and Tracking of Faces and Gestures in Real-time Systems, http://ronicslabz.com/welcome/pdf/RL Project Circuit 1405919659.pdf.

[10] Loeding Barbara, Sarkar Sudeep, "Automated extraction of signs from continuous sign language sentences using iterated condition modes", IEEE Computer Society Conference on Computer Vision and Pattern Recognition. June 2009.

[11] Geetha priya J and Srinivasan A, "A new framework for real-time hand gesture detection and recognition\| in International conference on information and network technology (ICINT 2012), IPCSIT vol. 37 (2012) C (2012) IACSIT Press, Singapore 2012.

[12] Ghosh, D.K. , Ari, S. -A Static Hand Gesture Recognition Algorithm Using K-Mean Based Radial Basis Function", Neural Network\| in Information, Communications and Signal Processing (ICICS) 2011, 8th International Conference on 13-16 Dec. 2011.

[13] Amitkumar Shinde and Ramesh M. Kagalkar," Advanced Marathi Sign Language Recognition using Computer Vision", International Journal of Computer Applications, (ISSN:0975 - 8887), Volume 118, No. 13, May 2015.

[14] Rashmi. B. Hiremath and Ramesh. M. Kagalkar, "Methodology for Sign Language Video Interpretation in Hindi Text Language", International Journal of Innovative Research in Computer and Communication Engineering, Volume. 4, Issue 5, May 2016.

[15] Rashmi. B. Hiremath and Ramesh. M. Kagalkar,"Sign Language Video Processing for Text Detection in Hindi Language", International Journal of Recent Contributions from Engineering, Science and IT, Volume 4, No 3, 2016.

[16] Rashmi. B. Hiremath and Ramesh. M. Kagalkar" A Methodology for Sign Language Video Analysis and Translation into Text in Hindi Language", CiiT International Journal of Fuzzy Systems, Volume 8, No 5, 2016.

[17] Ramesh M. Kagalkar and Dr. S.V. Gumaste,"Review Paper: Detail Study for Sign Language Recognization Techniques" CiiT international journal of Digital Image Processing, Volume 8, No 3,2016.

[18] Ramesh M. Kagalkar, Dr. Nagaraj H.N and Dr. S.V Gumaste, "A Novel Technical Approach for Implementing Static Hand Gesture Recognition ”, International Journal of Advanced Research in Computer and Communication Engineering, Voume 1. 4, Issue 7, July 2015.

[19] Ramesh M. Kagalkar, Dr. Nagaraj H.N," New Methodology for Translation of Static Sign Symbol to Words in Kannada Language ", International Journal of Computer Applications (ISSN: 0975 - 8887) Volume 121 - No.20, July 2015.

[20] Ramesh M. Kagalkar and S.V Gumaste, "Gradient Based Key Frame Extraction for Continuous Indian Sign Language Gesture Recognition and Sentence Formation in Kannada Language: A Comparative Study of Classifiers", International Journal of Computer Sci- 
Paper-ANFIS Based Methodology for Sign Language Recognition and Translating to Number in Kan...

ences and Engineering, Volume-04, Issue-09, Page No (1-11), Sep -2016, E-ISSN: 23472693.

[21] G. Joshi, J. Sivaswamy, "A simple scheme for contour detection", 2004 pages 236-242, https://researchweb.iiit.ac.in/ gopal/pub/visapp06.pdf.

[22] K. R. Linstrom and A.J. Boye. "A neural network prediction model for a psychiatric application" in International Conference on Computational Intelligence and Multimedia Applications, pp. 36-40, 2005.

[23] J. Yamato, J. Ohya, and K. Ishii, "Recognizing human action in time sequential images using hidden Markov model," in Proc. IEEE Int. Conf. Computer. Vis. Pattern Recogn., Champaign, IL, 1992, pp. 379-385.

[24] Rung-Huei Liang Ming Ouhyoung, "A Real-time continuous alphabetic sign language to speech conversion VR system" in Communications \& Multimedia Lab., Computer Science and Information Engineering Dept., National Taiwan University, Taipei, Taiwan.

[25] Rung-Huei Liang Ming Ouhyoung, "A Real-time continuous gesture recognition system for sign language" in National Taiwan University, Taipei, Taiwan.

\section{Authors}

Ramesh M. Kagalkar was born on Jun 1st, 1979 in Karnataka, India and presently working as an Assistant. Professor, Department of Computer Engineering, Dr. D Y Patil School of Engineering and Technology, Charoli, B.K.Via Lohegaon, Pune, Maharashtra, India. He has 14.5 years of teaching experience at various institutions. He is a Research scholar in Visveswaraiah Technological University, Belgaum, He had obtained M.Tech (CSE) Degree in 2006 from VTU Belgaum and He received BE (CSE) Degree in 2001 from Gulbarga University, Gulbarga Karnataka, India. He is the author of two text book; 1.Advance Computer Architecture, 2. The Swift Practical Approach of Learning C-Programming in LAP LAMBERT Academic Publishing, Germany (Available in online) and One of his research article A Novel Approach for Privacy Pre- serving has been consider as text in LAP LAMBERT Academic Publishing, Germany (Available in online). He is waiting for submission of two research articles for patent right. He has published more than 35 research papers in International Journals and presented few of there in international conferences. His main research interest includes Image processing, Gesture recognition, Speech processing, Voice to sign language and CBIR (rameshvtu10@gmail.com).

Dr. S.V Gumaste was born on 02 April 1968 in Karnataka, India and presently working as a Professor \& Head, Computer Engineering, Computer Engineering, R. H. Sapat College of Engineering, Nashik, Maharashtra, India. He has 22.5 years of teaching experience at various institutions. He received $\mathrm{Ph} . \mathrm{D}$ degree from Sant Gadge Baba Amravati University, Amravati in 2015. He obtained M.E (CSE) Degree in 2007 from Prof. Ram Meghe Institute of Technology \& Research, Badnera, Amravati. (Sant Gadge Baba Amravati University, Amravati, Maharashtra) and He received BE (CSE) Degree in 1992 from BLDE College of Engineering \& Technology, Bijapur. (Karnataka University, Dharwar). He is the author of 03 Books such as fundamentals of C Programming, Exel Publication, Amravati. (In August, 2005), C Programming with 150 solved programs, Exel Publication, Amravati. (In January, 2005) and Basics of C Programming: A Practical Approach, Jai Publication, Pusad. (In Au- 
gust, 2004). He has published more than 12 research papers in International Journals and presented 12 papers in international conferences. His main research interest includes networking, image processing and speech processing. He is the life Membership with Professional Bodies of LM-20507, Indian Society for Technical Education, New Delhi, Institutional Member (N0046703), Computer Society of India, Mumbai (2008-09) and (MH/222/1645), All India Federation of University \& College Teachers' Organisations, Hyderabad (AIFUCTO) (svgumaste@ gmail • com )

Article submitted 21 January 2017. Published as resubmitted by the authors 03 March 2017. 